وقائع المؤتمر العلمي الحادي عشر- كلية الطب البيطري 20- 27: 2012

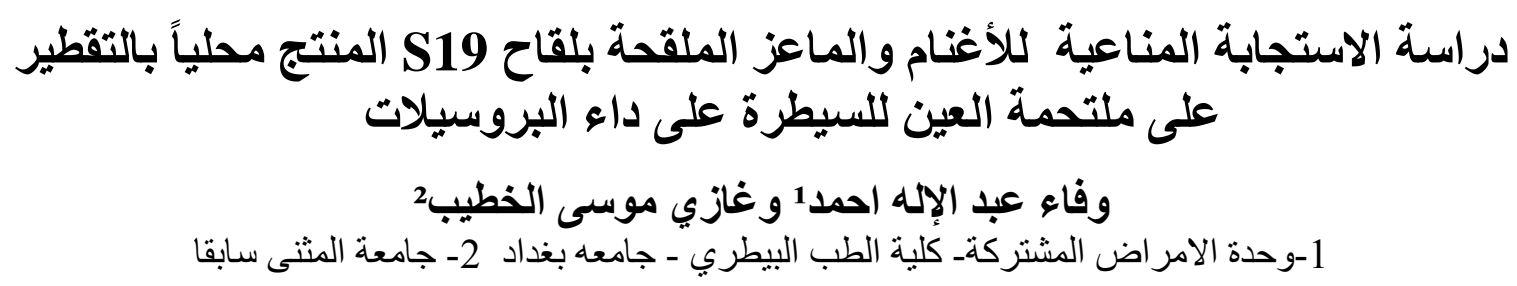

\title{
الخلاصة
}

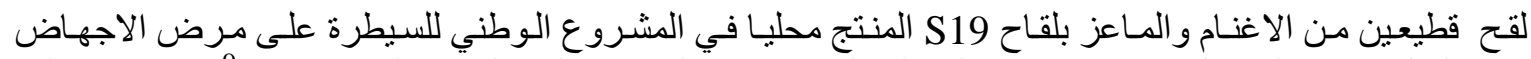

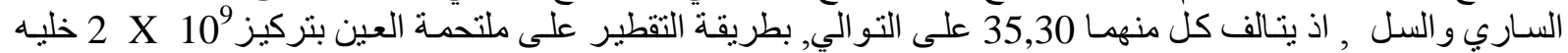

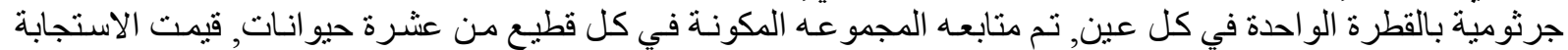

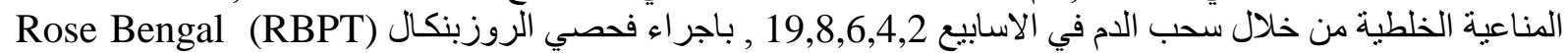

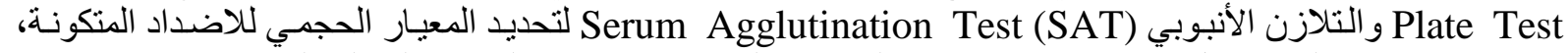

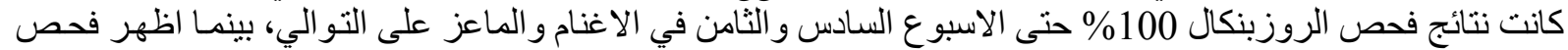

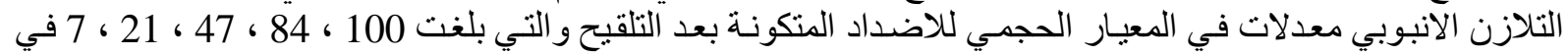

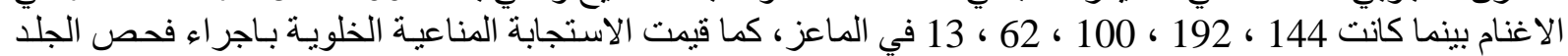

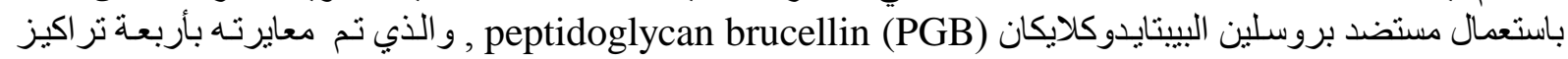

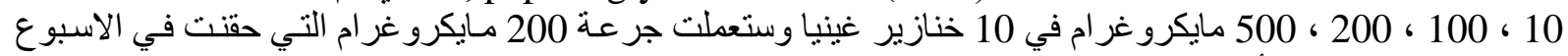

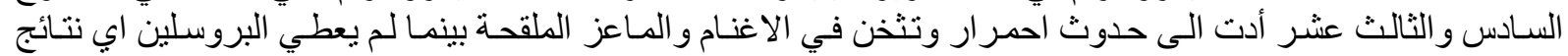

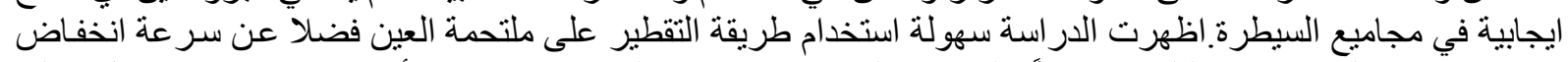

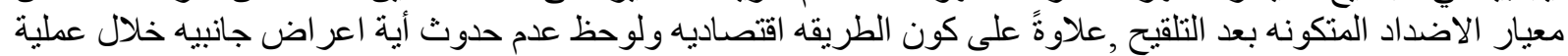

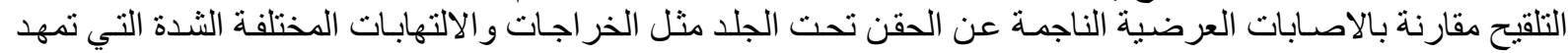

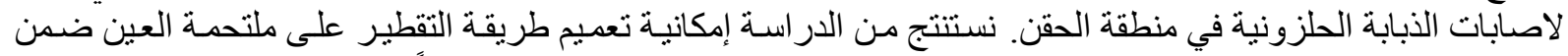

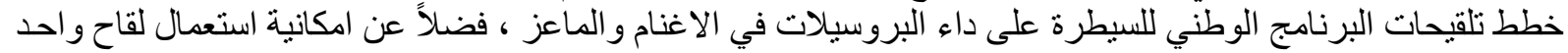
مشترك لتلقيح الحيو انات الحقلية ضد الإندابة للإطرة بهذا الداء.

\section{Study the Immune Response of Conjunctival Vaccinated Sheep and Goats with Locally Produced S19 Vaccine for Controlling the Brucellosis}

\author{
Waffa A.A. ${ }^{1}$ and G.M. AL-Khatib ${ }^{2}$ \\ 1-College of Veterinary Medicine - Baghdad University 2- AL-Muthanna University \\ Previously.
}

\section{Summary}

Two flocks of sheep and goats (30 and 35 animals) were vaccinated by locally produced S19 vaccine, each animal instilled with $2 \times 10^{9} \mathrm{cfu}$ in each drop conjunctively. From each flock, a group of 10 heads was used to study the immune response serologically at intervals 2 , $4,6,8$, and 19 week through blood samples using the rose bengal plate test (RBPT) and serum agglutination test (SAT) to determine Ab titres.

The results of RBPT were $100 \%$ positive till 6 and 8 weeks in sheep and goats respectively, while SAT showed means of Ab titer after vaccination 100, 84, 47, 21, and 7 in sheep while in goats reached 144, 192, 100, 62, and 13. Also, the cellular immune response was evaluated by prepared Peptidoglycan Brucellin (PGB), which was injected in 10 guinea pigs through 4 concentrations $10,100,200$, and $500 \mu \mathrm{g}, 200 \mu \mathrm{g}$ was used in brucellin test at the sixth and thirteenth week after vaccination, the antigen caused erythemia and induration in the vaccinated sheep and goats while the test give negative results in non-vaccinated animals.

The study revealed that the conjunctival route is more safe, easy, and economic, and also the rapid reduce of Abs titer compared with subcutaneous route. Also, it is noticed that there were no side effects in the conjunctival route comparatively with subcutaneous route such as inflammation, abscess, and screw worm in the worm infestation in the site of injection. 


\section{وقائع المؤتمر العلمي الحادي عشر- كلية الطب البيطري 20- 27: 2012}

The study concluded the possibility of using conjunctival route in the vaccination campaign for controlling brucellosis in sheep and goats and also possibility of using one vaccine for different field animals against brucellosis.

\section{المقدمة}

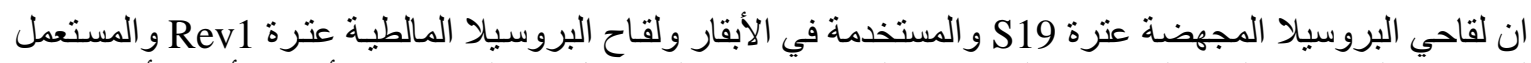

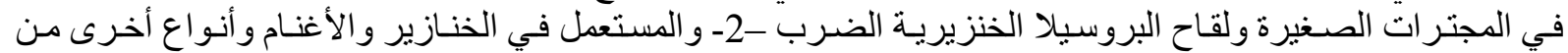

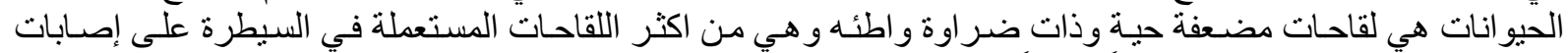

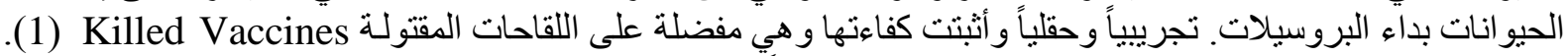

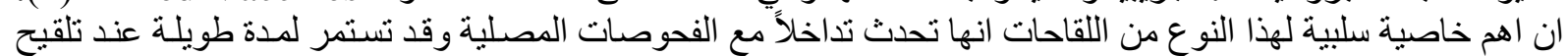

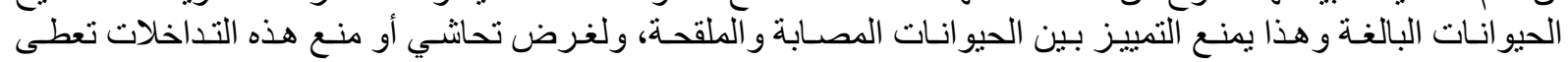

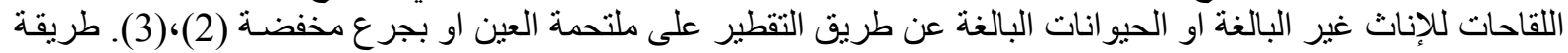

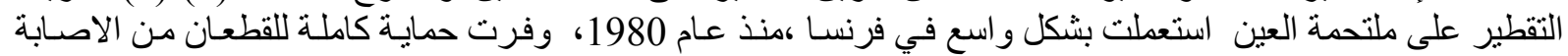

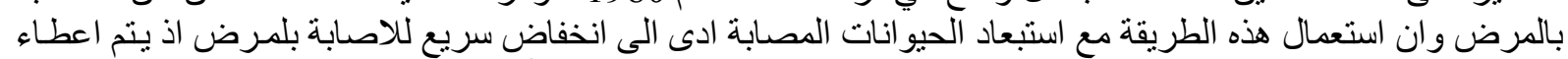

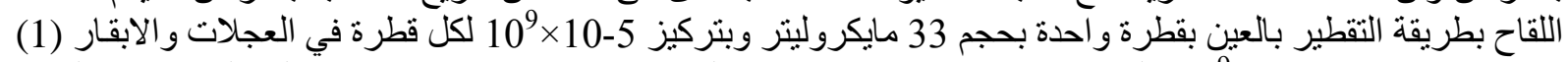

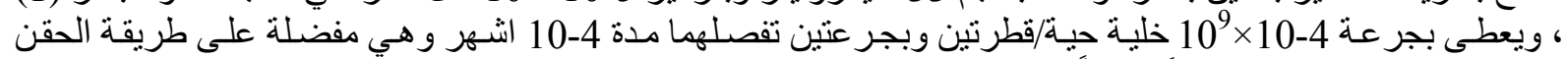

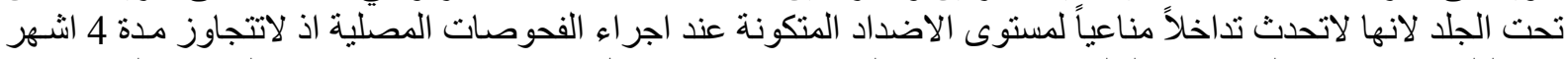

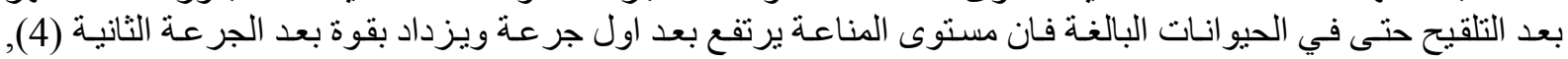

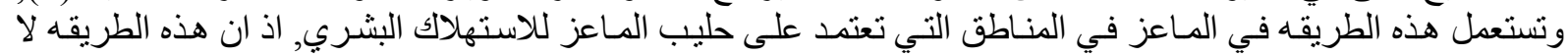

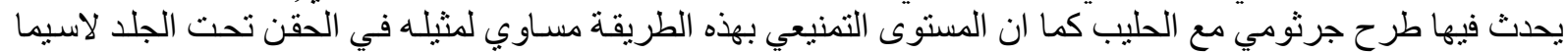

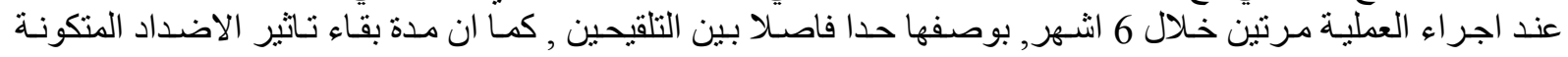

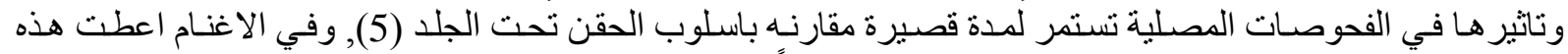

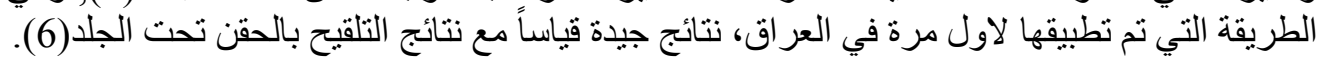

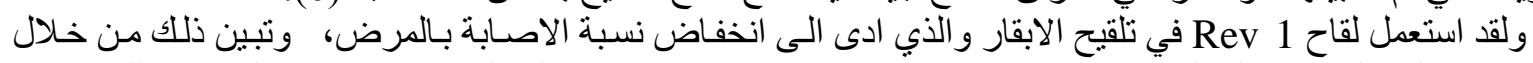

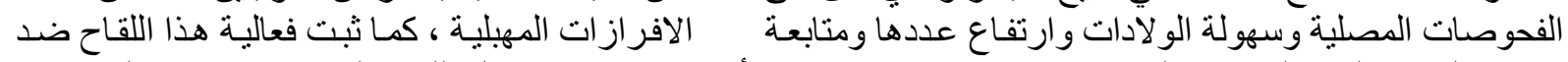

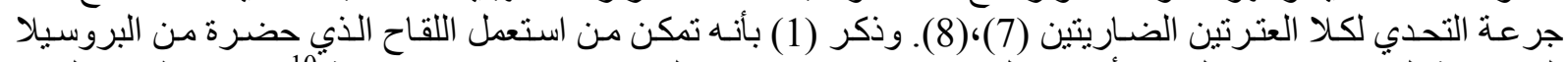

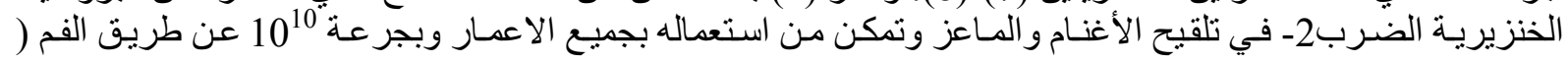

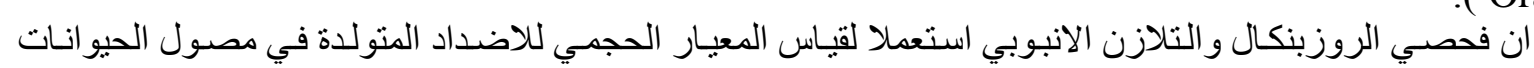

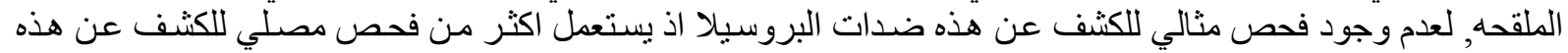

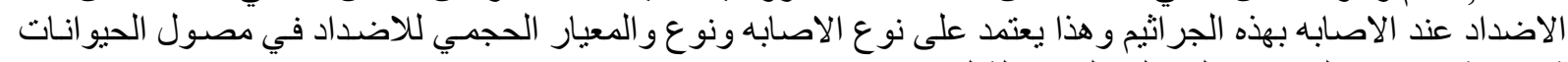

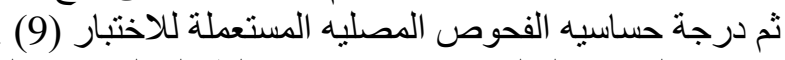

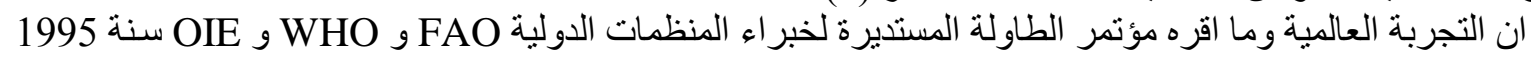

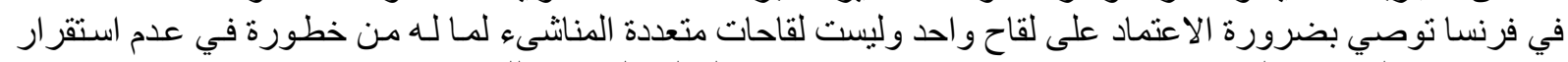

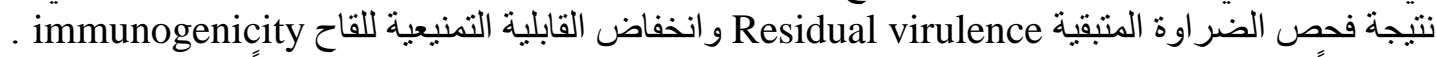

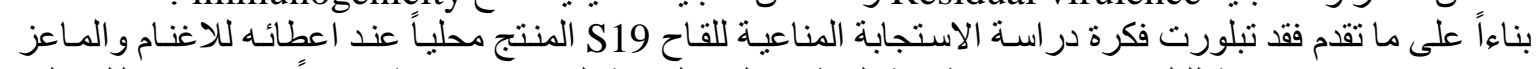

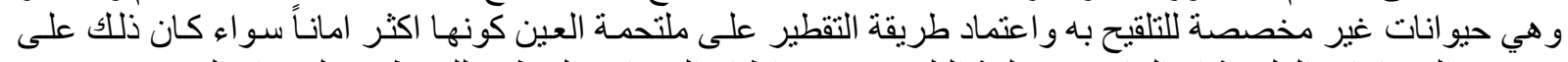

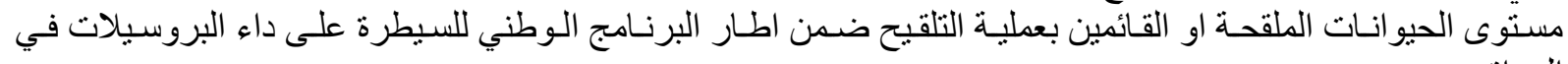

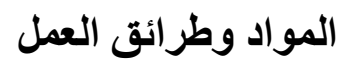

العر اق.

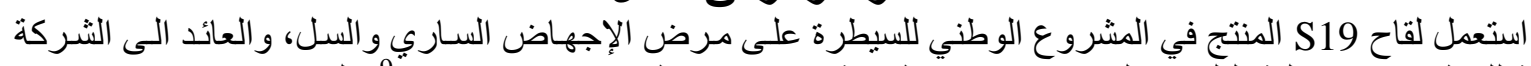

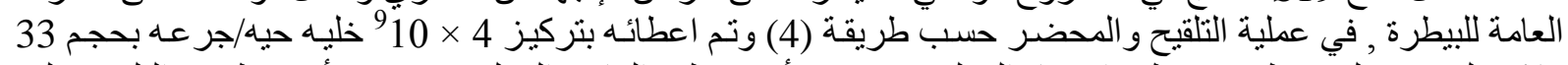

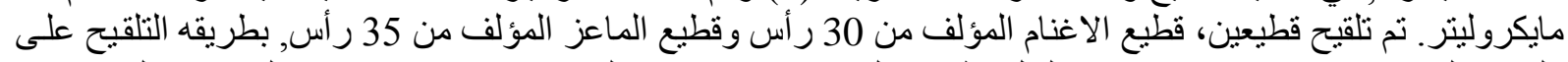

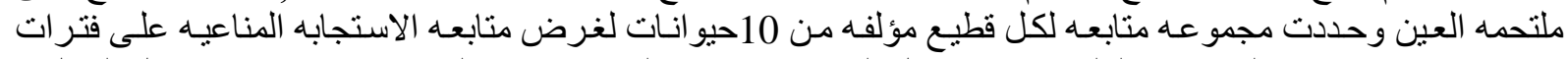

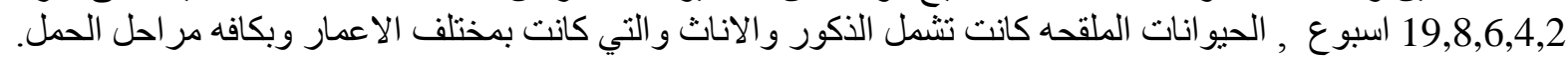

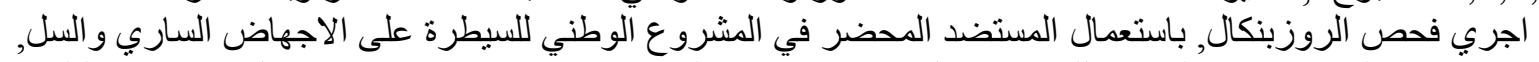

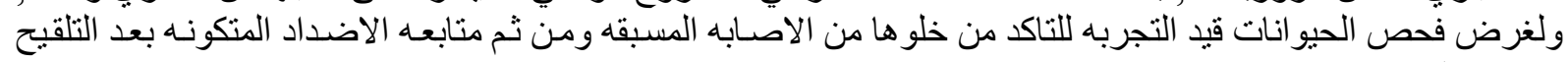

وحسب طريقة (4).

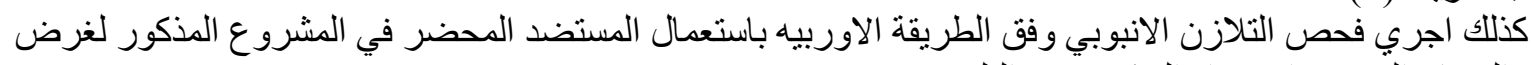

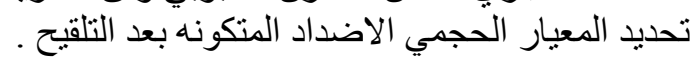

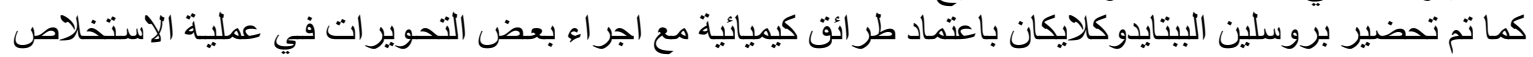

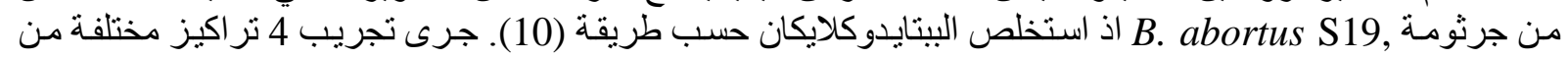




\section{وقائع المؤتمر العلمي الحادي عشر- كلية الطب البيطري 20- 27: 2012}

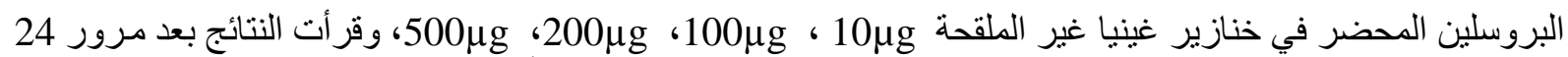

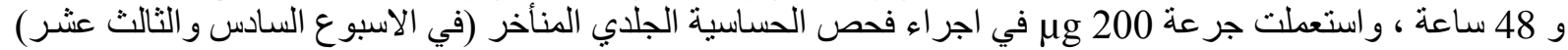

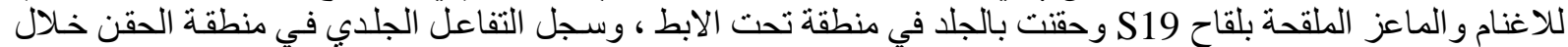
24 ، 48 ساعة بعد الحقن من خلال قر اعة قطر الاحمر ار وتثنخ الجلد بوساطة المسطرة المعدنية.

\section{النتائج}

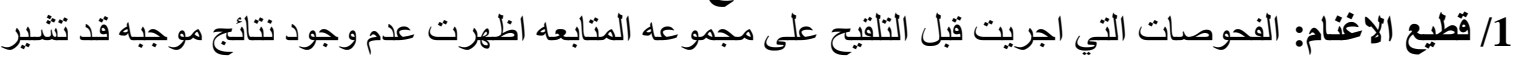

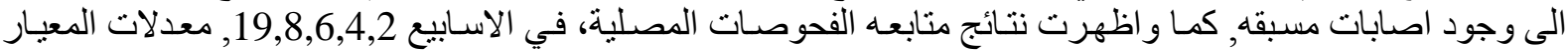

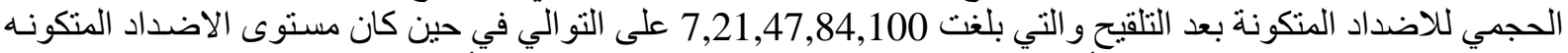

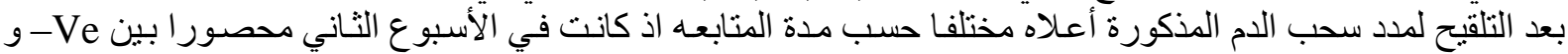

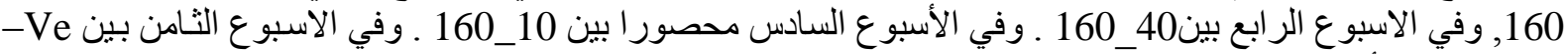

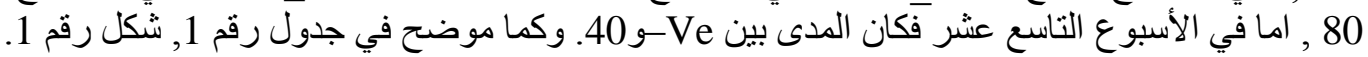

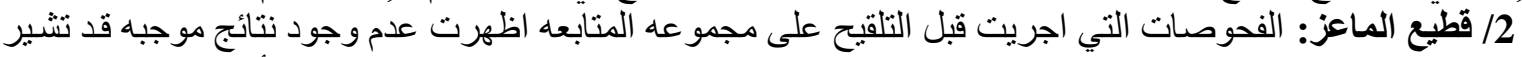

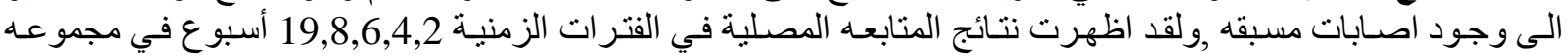

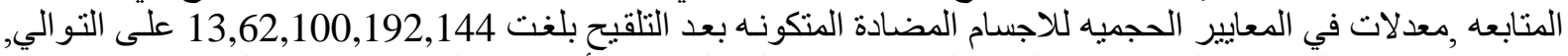

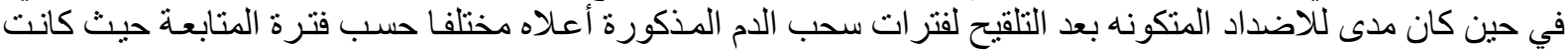

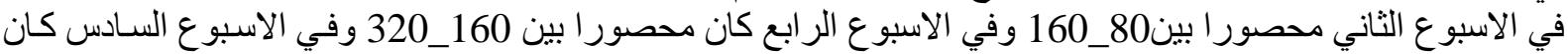

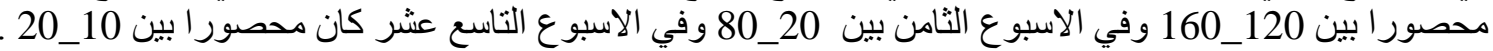

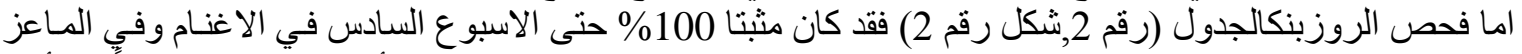

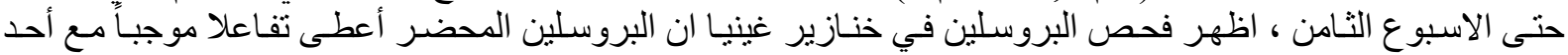

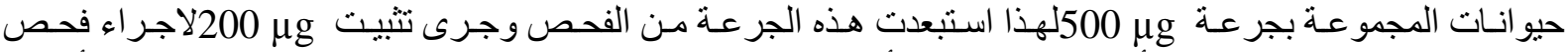

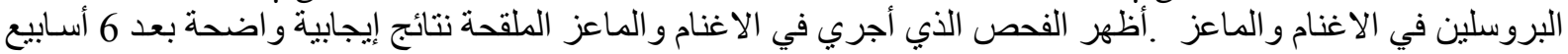

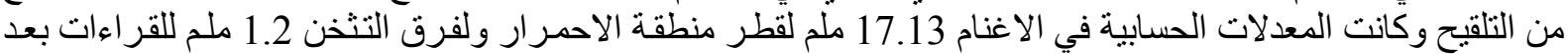

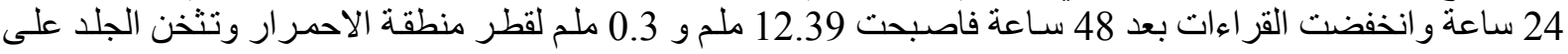

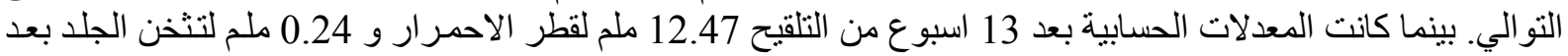

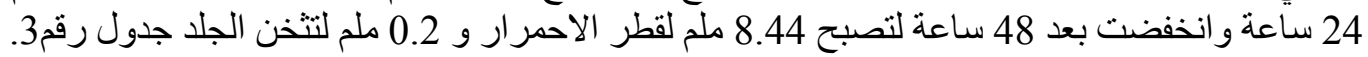

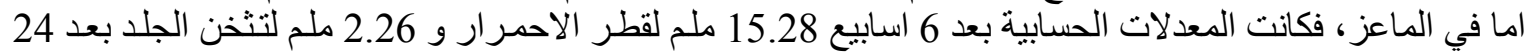

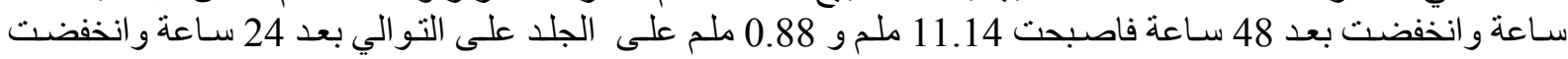
لتصبح 3.74 ملم و 0.22 ملم بعد 48 ساعة على التو الي جدول رقم4.

جلول رقم (1) :نتائج الفحوصات المصلية للاغنام الملقحة بلقاح S19 بطريقة التقطير بالعين

\begin{tabular}{|c|c|c|c|c|c|c|c|c|c|c|c|}
\hline \multirow{4}{*}{$E$} & \multirow{4}{*}{ قالروزبنكال } & \multicolumn{10}{|c|}{ اســــابيع سحــب الـــــم } \\
\hline & & \multicolumn{2}{|c|}{2} & \multicolumn{2}{|c|}{4} & \multicolumn{2}{|c|}{6} & \multicolumn{2}{|c|}{8} & \multicolumn{2}{|c|}{19} \\
\hline & & فصص & فحص & فحص & فحص & فحص & فحص & فحص & فحص & فحص & فحص \\
\hline & & الزروزبنكال & الالتبلازبي & الروزبنكال & الالتبونبي & الروزينكال & الأنتلازبي & الروزبنكال & الانبوبي & الروزينكال & الالتبوبي \\
\hline 1 & -ve & + & 80 & + & 80 & + & 80 & + & 20 & -ve & - \\
\hline 2 & -ve & + & 160 & + & 160 & + & 160 & + & 80 & + & 40 \\
\hline 3 & -ve & + & 160 & + & 80 & + & 80 & + & 40 & + & 10 \\
\hline 4 & -ve & + & 40 & + & 80 & + & 20 & -ve & -ve & -ve & -ve \\
\hline 5 & -ve & + & 80 & + & 40 & + & 40 & -ve & 10 & -ve & -ve \\
\hline 6 & -ve & + & 160 & + & 160 & + & 40 & + & 20 & + & 10 \\
\hline 7 & -ve & + & 80 & + & 40 & + & 10 & -ve & -ve & -ve & -ve \\
\hline 8 & -ve & + & 160 & + & 80 & + & 40 & -ve & 10 & -ve & -ve \\
\hline 9 & -ve & + & -ve & + & 80 & + & 40 & + & 20 & + & 10 \\
\hline $\begin{array}{l}1 \\
0\end{array}$ & -ve & + & 80 & + & 40 & + & 40 & -ve & 10 & -ve & $-v e$ \\
\hline
\end{tabular}


وقائع المؤتمر العلمي الحادي عشر- كلية الطب البيطري 20- 27: 2012

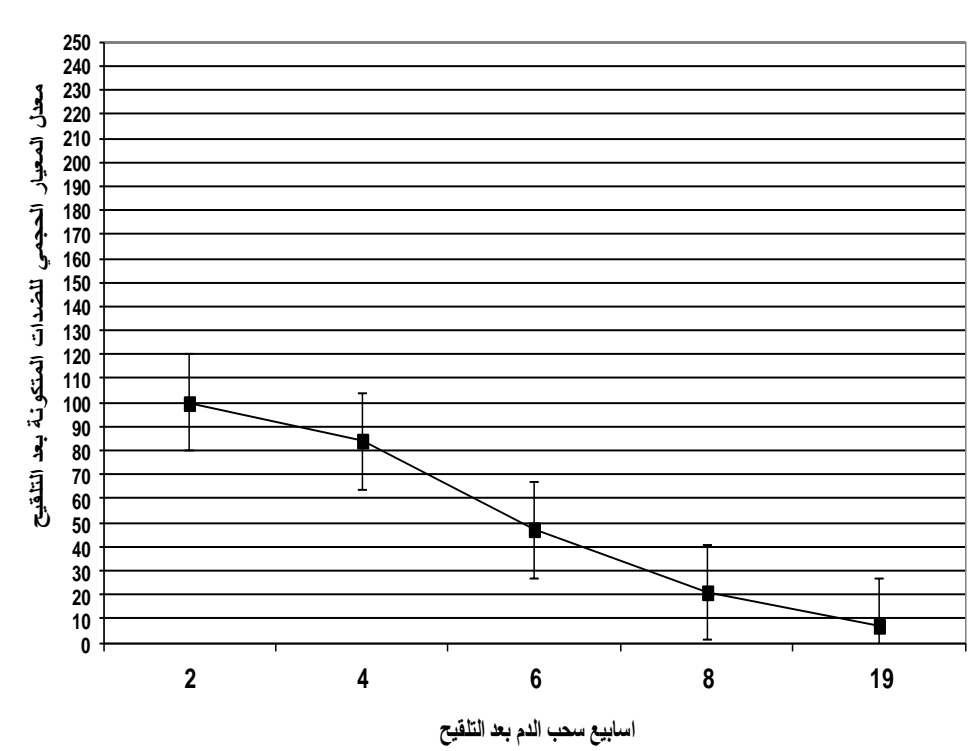

\begin{tabular}{|c|c|c|}
\hline المجمي للاضداد المتكونة & الحيو انات & الاسبوع \\
\hline 100 & 10 & 2 \\
\hline 84 & 10 & 4 \\
\hline 47 & 10 & 6 \\
\hline 21 & 10 & 8 \\
\hline 7 & 10 & 19 \\
\hline
\end{tabular}

الثكل رقم (1) : معدل معيار ومدى تكوين للاضداد في الاغنام الملقحة بلقاح S19 بطريقة التقطير على ملتحمة العين باستعمال فحص التلازن الانبوبي للمصل للافل فئل

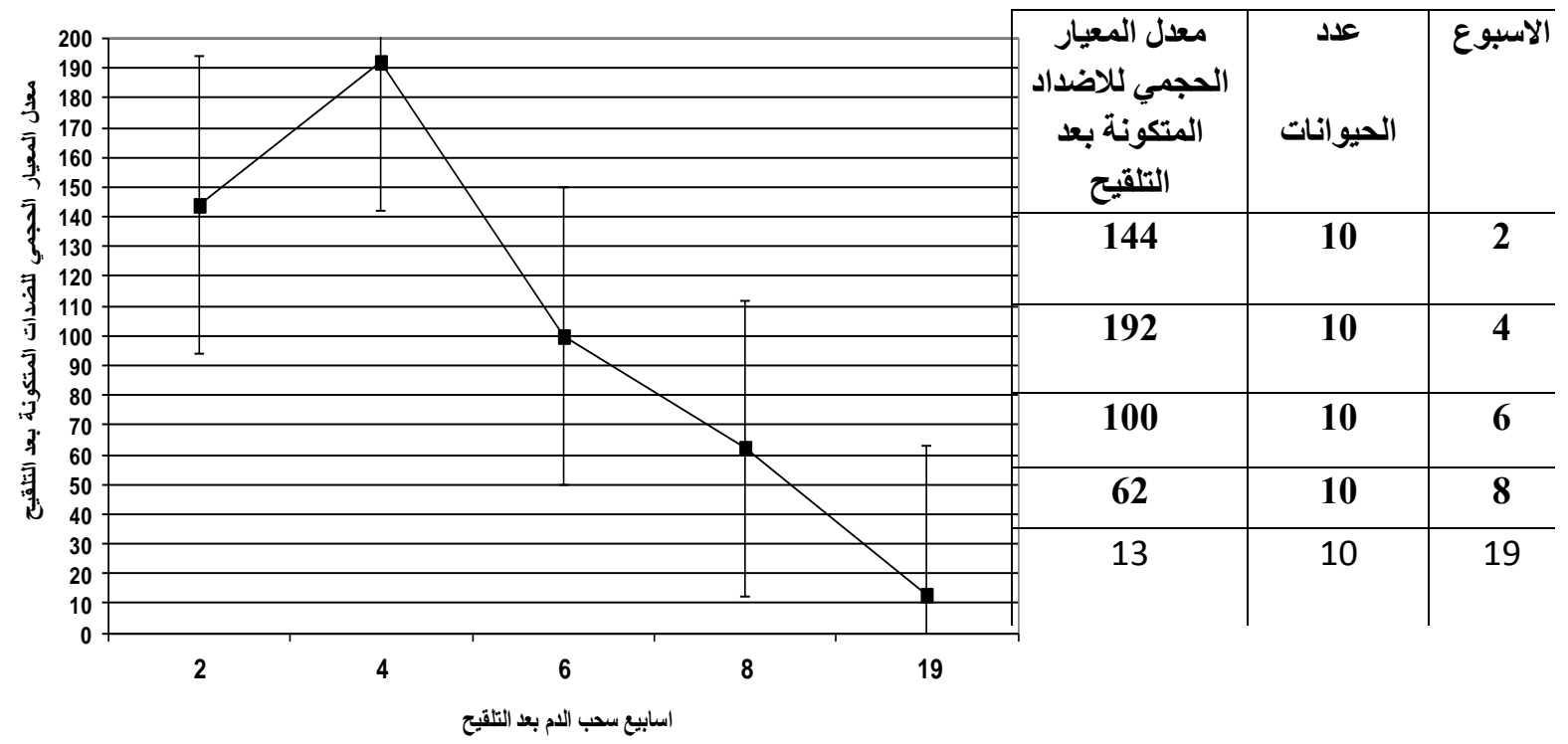

الثكل رقم (2) : معدل معيار ومدى تكوين الضدات في الماعز الملقح بلقاح S19 بطريقة التقطير على ملتحمة العين باستعمال فحص التلازن الانبوبي للمصل 
وقائع المؤتمر العلمي الحادي عشر- كلية الطب البيطري 20- 27: 2012

جدول رقم (2) : نتائج الفحوصات المصلية للماعز الملقحة بلقاح S19 بطريقة التقطير على ملتحمة العين

\begin{tabular}{|c|c|c|c|c|c|c|c|c|c|c|c|}
\hline \multicolumn{12}{|c|}{ اسنابيع سحب الام } \\
\hline \multirow{2}{*}{$\underline{E}$} & \multirow{2}{*}{ فبل التثلثينكال } & \multicolumn{2}{|l|}{2} & \multicolumn{2}{|l|}{4} & \multicolumn{2}{|c|}{6} & \multicolumn{2}{|l|}{8} & \multicolumn{2}{|c|}{19} \\
\hline & & فحصزينكال & فلالنصوبي التلازن & فرصنيكال & فالانبوبي التلازن & فحصنيكال & فحص الآنيوبيلازن & فرصزينكال & فحص الانبوبي & فروزينكال & التلازلن \\
\hline 1 & -ve & + & 160 & + & 320 & + & 160 & + & 80 & + & 20 \\
\hline 2 & -ve & + & 160 & + & 160 & + & 160 & + & 80 & + & 10 \\
\hline 3 & -ve & + & 80 & + & 320 & + & 160 & + & 80 & + & 20 \\
\hline 4 & -ve & + & 160 & + & 160 & + & 160 & + & 80 & + & 20 \\
\hline 5 & -ve & + & 160 & + & 160 & + & 80 & + & 40 & - & 10 \\
\hline 6 & -ve & + & 80 & + & 160 & + & 20 & + & 20 & - & 10 \\
\hline 7 & -ve & + & 160 & + & 160 & + & 80 & + & 80 & + & 10 \\
\hline 8 & -ve & + & 160 & + & 160 & + & 40 & + & 40 & - & 10 \\
\hline 9 & -ve & + & 160 & + & 160 & + & 40 & + & 40 & - & 10 \\
\hline 10 & -ve & + & 160 & + & 160 & + & 80 & + & 80 & - & 10 \\
\hline
\end{tabular}

جدول رقم ( 3) :نتائج اختبار فحص الحساسية الجلدية مستضد البروسلين في الاغنام الملقحة بلقاح S19 بطريقة التقطير على ملتحمة العين بعد 6 و 13 اسبوع فيوع من التلقيح

\begin{tabular}{|c|c|c|c|c|c|c|c|c|c|c|c|c|}
\hline \multirow[t]{3}{*}{ التسلسل } & \multicolumn{6}{|c|}{ الاسبوع السادس } & \multicolumn{6}{|c|}{ الاسبوع الثالث عشر } \\
\hline & \multirow[t]{2}{*}{ بنكال } & \multirow{2}{*}{ مستركيز } & \multicolumn{2}{|c|}{ التفاعل خلال 24} & \multicolumn{2}{|c|}{ التفاعل خلال 48} & \multirow[t]{2}{*}{ الروزل } & \multirow{2}{*}{ مستركيز } & \multicolumn{2}{|c|}{ التفاعل خلال 24 سـاعة } & \multicolumn{2}{|c|}{ التفاعل خلال 48} \\
\hline & & & $\begin{array}{c}\text { منطقة } \\
\text { الاحمرار } \\
\text { mm }\end{array}$ & الجثل & الاحمرا & الجلد & & & $\begin{array}{c}\text { منطقة } \\
\text { الاحمرار } \\
\text { mm }\end{array}$ & $\begin{array}{l}\text { الجلدن } \\
\text { mm }\end{array}$ & $\begin{array}{c}\text { قاحمرار } \\
\text { mm }\end{array}$ & الجلد \\
\hline .1 & + & $200 \mu \mathrm{g}$ & 17.76 & 1.55 & 12.75 & 0.2 & + & $200 \mu \mathrm{g}$ & 14.6 & 0.3 & 10.2 & 0.1 \\
\hline .2 & + & $200 \mu \mathrm{g}$ & 14.81 & 0.3 & 10.5 & $-\mathrm{Ve}$ & + & $200 \mu \mathrm{g}$ & 12.5 & 0.2 & 9.5 & $-\mathrm{Ve}$ \\
\hline .3 & + & $200 \mu \mathrm{g}$ & 14.3 & 2.05 & 10.3 & 0.4 & + & $200 \mu \mathrm{g}$ & 9.1 & 0.2 & 5.1 & $-\mathrm{Ve}$ \\
\hline .4 & + & $200 \mu \mathrm{g}$ & 24.7 & 1.5 & 19.5 & 0.6 & + & $200 \mu \mathrm{g}$ & 12.46 & 0.2 & 8.2 & $-\mathrm{Ve}$ \\
\hline .5 & + & $200 \mu \mathrm{g}$ & 14.1 & 0.6 & 8.9 & 0.1 & + & $200 \mu \mathrm{g}$ & 13.7 & 0.3 & 9.2 & $-\mathrm{Ve}$ \\
\hline & $-\mathrm{Ve}$ & PBS & VE- & $-\mathrm{Ve}$ & $-\mathrm{Ve}$ & $-\mathrm{Ve}$ & $-\mathrm{Ve}$ & PBS & $-\mathrm{Ve}$ & $-\mathrm{Ve}$ & $-\mathrm{Ve}$ & $-\mathrm{Ve}$ \\
\hline & $-\mathrm{Ve}$ & PBS & VE- & $-\mathrm{Ve}$ & $-\mathrm{Ve}$ & $-\mathrm{Ve}$ & -Ve & PBS & -Ve & - Ve & $-\mathrm{Ve}$ & $-\mathrm{Ve}$ \\
\hline الحسابي & & $200 \mu \mathrm{g}$ & 17.13 & 1.2 & 12.39 & 0.3 & & & 12.47 & 0.24 & 8.44 & 0.2 \\
\hline
\end{tabular}


وقائع المؤتمر العلمي الحادي عشر- كلية الطب البيطري 20- 27: 2012

جدول رقم ( 4) :نتائج اختبار فحص الحساسية الجلدية مستضد البروسلين في المـاعز الملقحة بلقاح S19 بطريقة التقطير بالعين بعد 6 و 13 اسبوع التبوع من التلقيح

\begin{tabular}{|c|c|c|c|c|c|c|c|c|c|c|c|c|}
\hline \multirow[t]{3}{*}{ التسلسل } & \multicolumn{6}{|c|}{ الاسبوع السادس } & \multicolumn{6}{|c|}{ الاسبوع الثالث عشر } \\
\hline & \multirow[t]{2}{*}{ البكال } & \multirow{2}{*}{ نوع وتركيز } & \multicolumn{2}{|c|}{ التفاعل خلال 24 ساء } & \multicolumn{2}{|c|}{ التفاعل خلال 48 ساء } & \multirow[t]{2}{*}{ الروزبنك } & \multirow{2}{*}{ منتركيز } & \multicolumn{2}{|c|}{ التفاعل خلال 24 ساء } & \multicolumn{2}{|c|}{ التفاعل خلال 48 ألماعة } \\
\hline & & & $\begin{array}{c}\text { منطقة } \\
\text { الاحمرار } \\
\text { mm }\end{array}$ & الجلد & $\begin{array}{r}\text { قطر الاحمرار } \\
\text { mm }\end{array}$ & الجلد & & & $\begin{array}{c}\text { قطظة } \\
\text { منطرةر } \\
\text { mm }\end{array}$ & الثلدن & $\begin{array}{r}\text { قاحمرار } \\
\text { mm }\end{array}$ & الجلد \\
\hline 1. & + & $200 \mu \mathrm{g}$ & 15.88 & 2.26 & 10.8 & 1.1 & + & $200 \mu \mathrm{g}$ & 5.5 & 0.02 & 2.5 & $-\mathrm{Ve}$ \\
\hline .2 & + & $200 \mu \mathrm{g}$ & 10.46 & 1.0 & 7.5 & 0.2 & + & $200 \mu \mathrm{g}$ & 12.0 & 1.0 & 7.5 & 0.2 \\
\hline .3 & + & $200 \mu g$ & 18.4 & 2.7 & 14.1 & 0.9 & + & $200 \mu \mathrm{g}$ & 9.6 & 1.9 & 6.6 & 0.9 \\
\hline .4 & + & $200 \mu \mathrm{g}$ & 10.9 & 0.84 & 7.5 & - -Ve & + & $200 \mu \mathrm{g}$ & 5.6 & 0.75 & 2.1 & $-V e$ \\
\hline .5 & + & $200 \mu \mathrm{g}$ & 20.8 & 4.5 & 15.8 & 2.2 & + & $200 \mu \mathrm{g}$ & -Ve & 0.7 & $-\mathrm{Ve}$ & - Ve \\
\hline & $-\mathrm{Ve}$ & PBS & $-\mathrm{Ve}$ & $-\mathrm{Ve}$ & $-\mathrm{Ve}$ & $-\mathrm{Ve}$ & $-\mathrm{Ve}$ & PBS & $-\mathrm{Ve}$ & - Ve & $-\mathrm{Ve}$ & - Ve \\
\hline & $-\mathrm{Ve}$ & PBS & $-\mathrm{Ve}$ & $-\mathrm{Ve}$ & -Ve & $-\mathrm{Ve}$ & $-\mathrm{Ve}$ & PBS & $-\mathrm{Ve}$ & $-\mathrm{Ve}$ & $-\mathrm{Ve}$ & $-\mathrm{Ve}$ \\
\hline المعاب المل & & & 15.28 & 2.26 & 11.14 & 0.88 & & & 6.54 & 0.87 & 3.74 & 0.22 \\
\hline
\end{tabular}

\section{المناقثة}

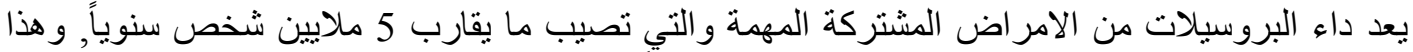

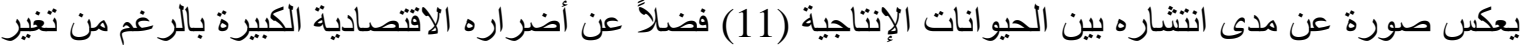

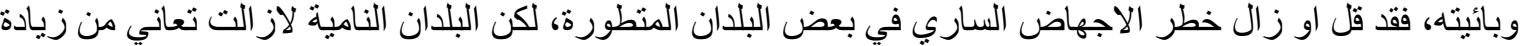

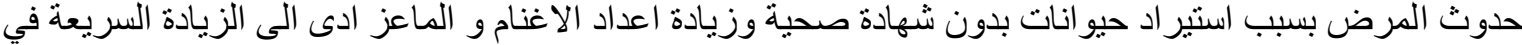

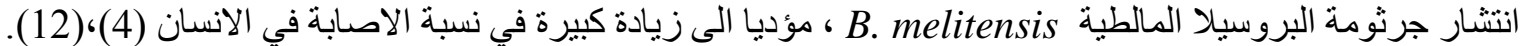

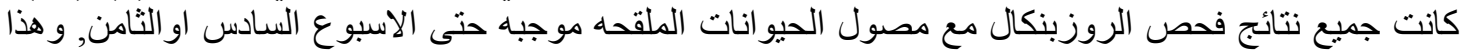

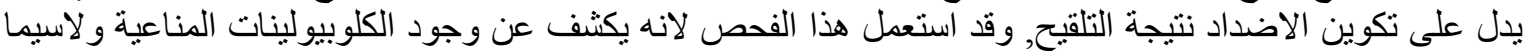

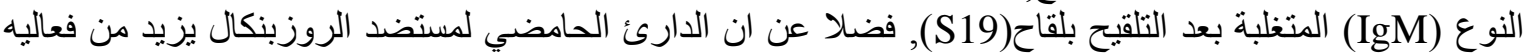

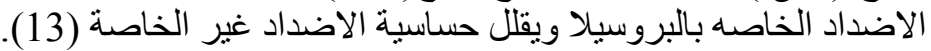

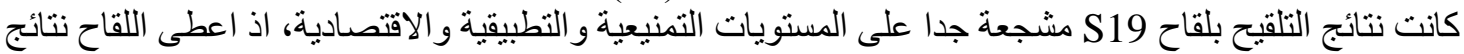

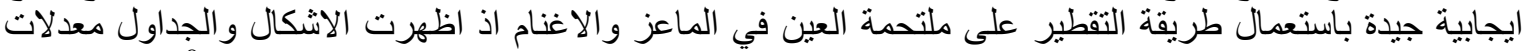

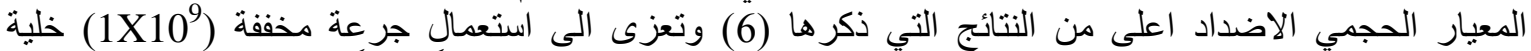

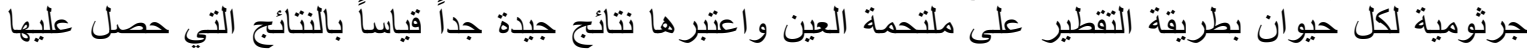

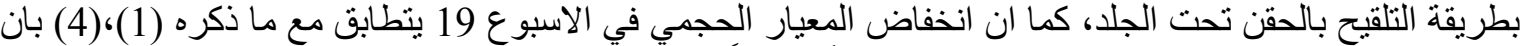

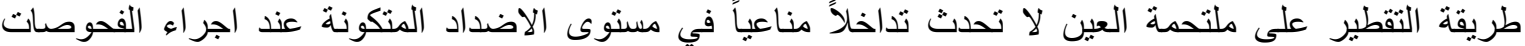

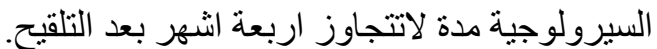

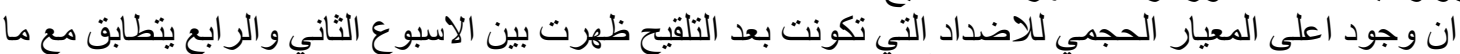

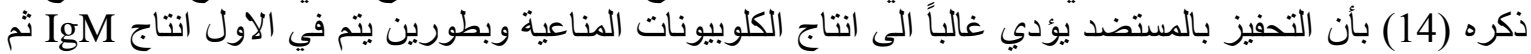

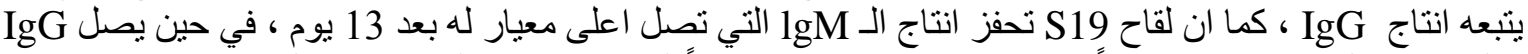

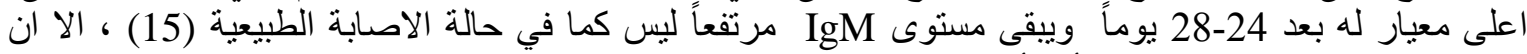

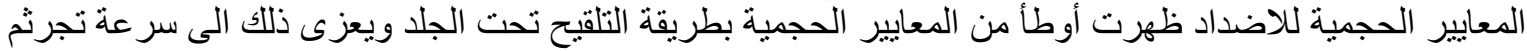

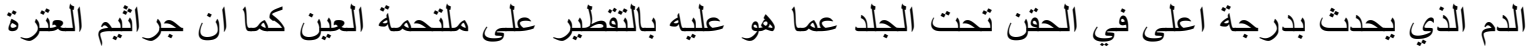

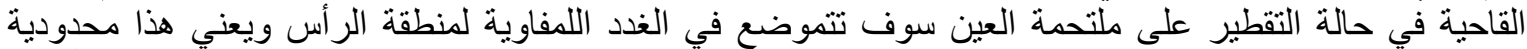

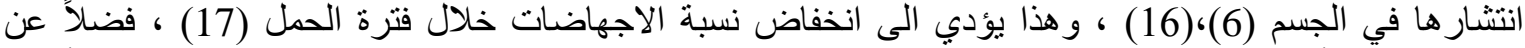

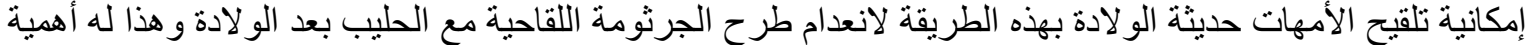

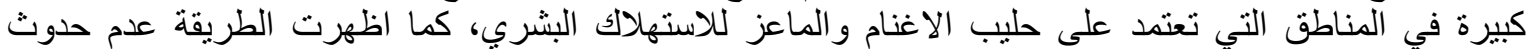

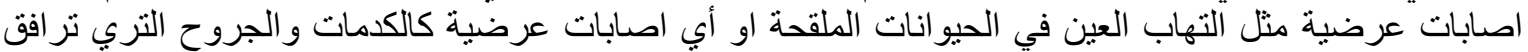

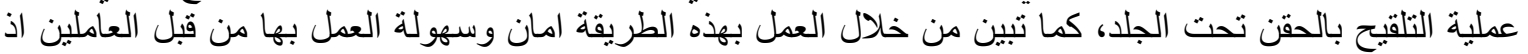

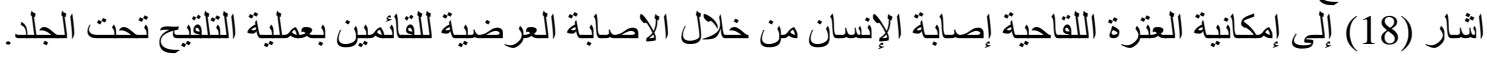




\section{وقائع المؤتمر العلمي الحادي عشر- كلية الطب البيطري 20- 27: 2012}

أضافت النتائج التي نوصلنا اليها معلومات جديدة حول اللقاح المنتج محلياً وهو ان اللقاح اعطى اعتى نتائج إيجابية

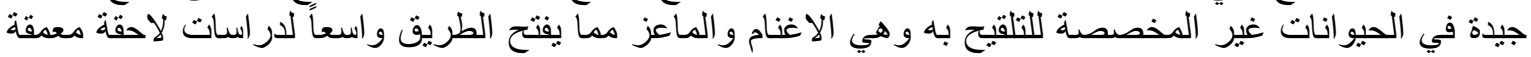

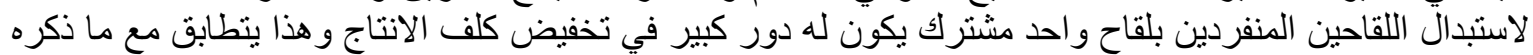

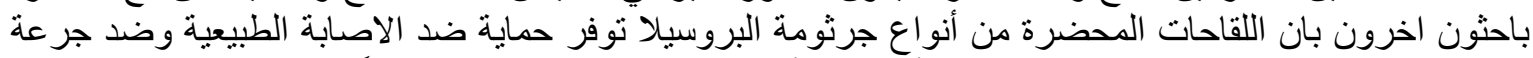

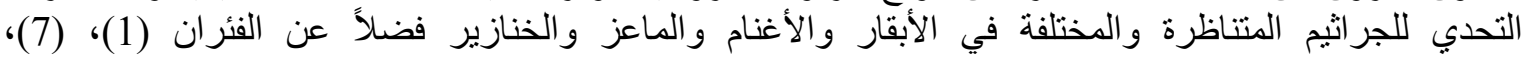

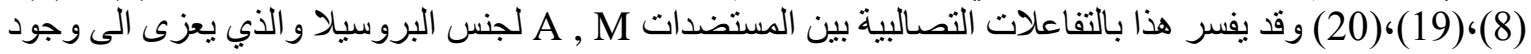
1,2 Linked Perosamine residues بصورة متغلبة في كلا المستضدين لذلك فان المصل المتعدد النسيلة يتفاعل التل بقوة دع كلا المستضدين (21).

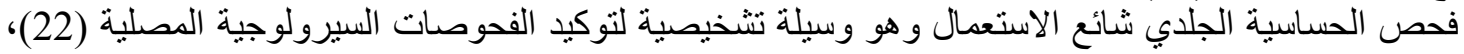

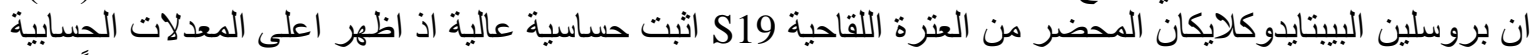

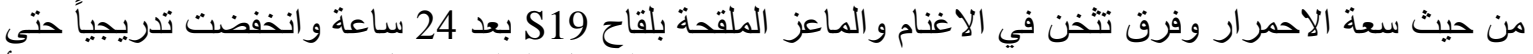

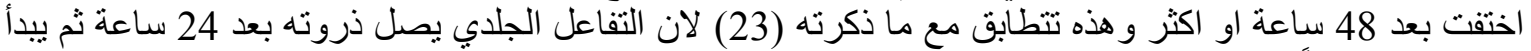

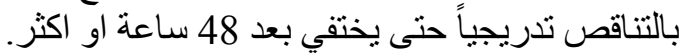

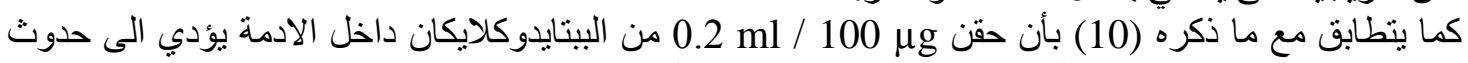

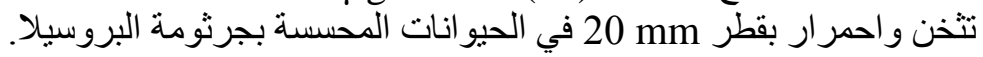

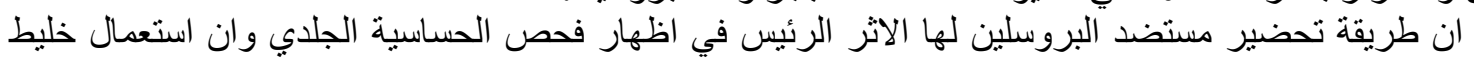

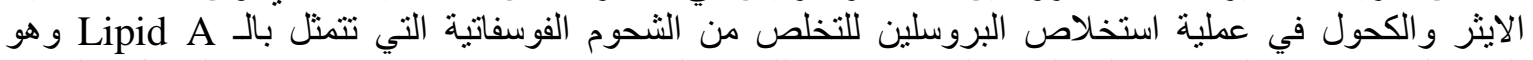

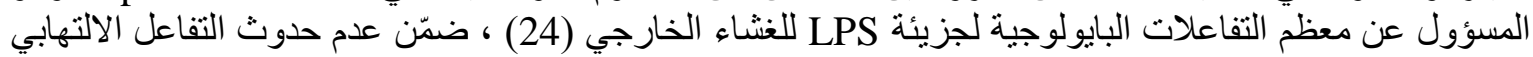

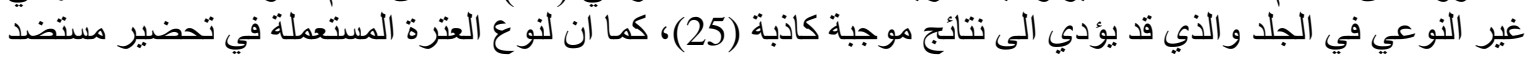

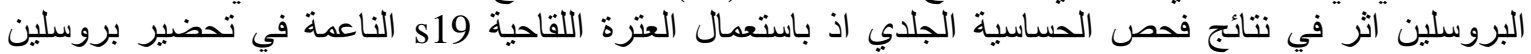

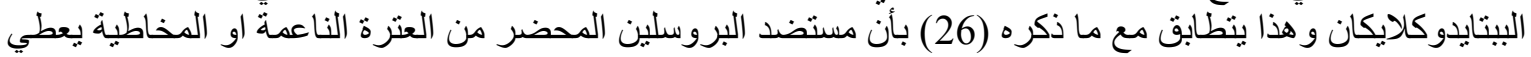

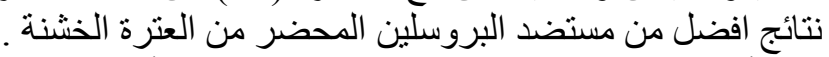

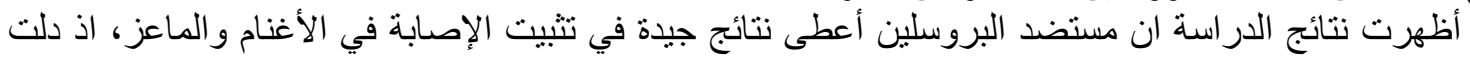

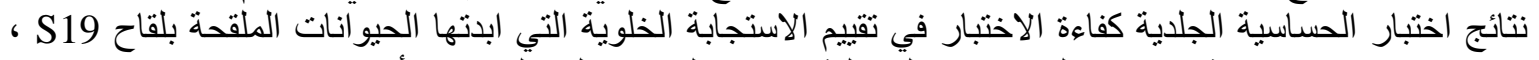

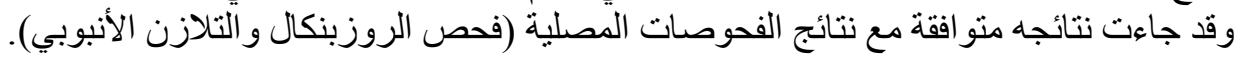

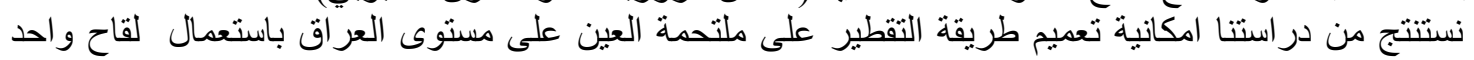
مشترك تلتيح الحيو انات الحقلية ضد الانية تعيب طرية بداء البروسيلات.

\section{المصادر}

1- Ryke, J. (1993). The QC of vaccines against Brucellosis FAO animal production and health paper: 116: 155-161.

2- Alton, G.G.; Corner, L.A. and Plaekett, P. (1980). Vaccination of pregnant cows with low doses of Brucella abortus st. 19 Vaccine. Aust. Vet. J., 56: 389.

3- Nicoletti, P. (1993). The Eradication of Brucellosis in Animals. Saudi Med. J., 14: 288 292.

4- Alton, G.G.; Jones, L.M.; Angus, R.D. and Verger, J.M. (1988). Techniques for the Brucellosis Laboratory. Institute national de La recherche agronomique 147, rue de I' university , 75007 Paris.

5- Alton, G.G. (1987). Control of Brucella melitensis infection in sheep and goats. A review. Trop. Anim. Hlth. Prod., 19: 65-74.

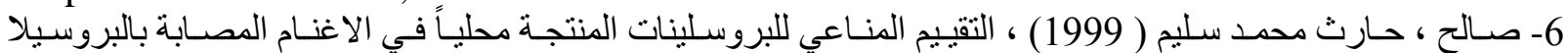

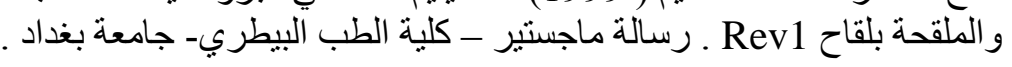

7- Kolar, J. (1995). Some experience from brucellosis control with Rev. I Vaccine in heavily infected country. Mongolia . FAO/ WHO/ OIE round table on the use of Rev.1 Vaccine in small ruminants and cattle . CNEVA, AL-FORT , France . Pp: $77-81$.

8- Banai, M.; Abramson, M.; lern-Mayer; Chechik, K.; Hoida, G.; Zami, O.; Bardenstein, S.; Cohen, A. and Davidson, M. (1995). Problems associated with the persistence and possible horizontal transfer of Brucella melitensis Rev.1 Vaccine in connection with serological surveillance in Israel. FAO/WHO/OIE : Round table in the use of Rev.1 vaccine in small ruminants and other CNEVA Alfort, France, (1995) PP: 69-76. 


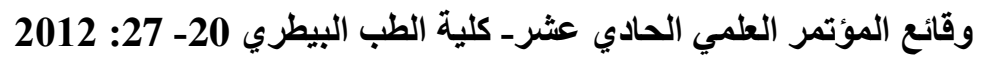

9- Morgan, B. (1977). Immunization Discussion in bovine brucellosis Ed, by Crawford, R,P, and Hidalgo, R,J, texas A and M, press, coll PP: 224-225.

10- (PIM) Protocol Institute Merieux (1986). Vaccine against brucellosis for human use . Lyon.

11- Atlas, R.M. (1995). Principles of Microbiology. $1^{\text {st }}$ Ed. University of Lonsvilli Kentucky, Mosbi, Yearbook Inc.: 648.

12- Joint FAO/WHO (1971). Expert committee on brucellosis. $5^{\text {th }}$ report WHO Tech. Rep. Ser. No., 464, Geneva.

13- Alton, G.G.; Jones, L.M. and Pietz, D.II. (1975). "Laboratory Technique". $2^{\text {nd }}$ Ed. Geneva WHO Monograph series No. 55 .

14- Nash, G.S.; Macdermott, R.P.; Schloemann, S.; Bertovich; O’Neal, J.; porter, L. and Kulczycki, A. (1990). Bovine IgG1. But not IgG2 bind to human B cells and inhibits Ab Secretion. Immunol., 69: 361-366.

15- Quinn, P.J.; Carter M.E.; Markey, B. and Carter, C.R. (1998). "Clinical Veterinary Microbiology". Mosby, Spain. Pp: 156-169

16- Verger, J.M. (1995). Efficacy and Advantages of the Rev.1 conjunctival vaccine against Br. melitensis infection, as evaluated in standard controlled conditions $\mathrm{FAO} / \mathrm{WHO} / \mathrm{OIE}$ round table on the use of the Rev.1 vaccine in small ruminants and cattle. CNEVA, Alfort. France, PP. 19-25.

17- Blasco, J.M. (1997). A review of the ewes of Brucella melitensis Rev1 vaccine in adult sheep and goat. Rev. Vet. Med. 31: 275-283.

18- Squaricone, S.; Maggi, P.; Lo, C.; Gennaro, M. and Carbonara, S. (1990). A case of humsn brucellosis caused by accidental injection of animal vaccine. J. Ital. Med., 12(1): 25-26.

19- Blasco, J.M.; Moriyon, I.; Marin, C. and Diaz, R. (1985). Evaluation of radial immunodiffusion test for differentiation infection from Rev1 vaccinated sheep in : Verger, J.M. and Plommet, M. 1985 Brucella melitensis a seminar in the CEC program of coordination of research on animal pathology, Martinez, Nijhof Publishers, Dordercht/ Boston / Lancaster. Pp: 147-154

20- Xie, X. (1986) orally administrated brucellosis vaccine, Brucella swis strain 2 vaccine. Vaccine, 4: 212 - 216.

21- Plackett, P.; Corner, L.A.; FiFis, T.; Radford, A.J. and Ripper, J.L. (1989). Discrimination between sheep antibodies to Brucella melitensis and to Brucella ovis. Vet. Microbiol., 20: 339-348.

22- Bercovich, Z.; Haagsma, J. and Ter-Laak, E.A. (1990). Use of delayed-type hypersensitivity test to diagnose brucellosis in calves born to infected dams. Vet. Q., 12: 231-237.

23- حمزة، اسيل محمد (2003). در اسة مقارنة لكفاءة البروسلينات المحضرة محليا في الكثف عن مرض البرة البروسيلوسز.

رسالة ماجستير - كلية الطب البيطري - جامعة بغداد.

24) Weintraub, A.; Larsson, B.E. and Lindberg, A.A. (1985). Chemical and immunochemical analysis of Bacteroides fragilis Lipopoly-saccharide. Infect. Immun., 49: 197-201.

25) Elberg, S.S. (1981). Rev 1 Brucella melitensis vaccine part II 1968-1980. Vet. Bull., 51 (2) : 67-73.

26) Bercovich, Z.; Eger, A.; Dekker, T. and Haagsma, J. (1995). Production of Brucella antigens and evaluation of their biological activity in guinea pigs. Bioassay J. Vet. Med. B., 42: 19-27. 